\title{
INOCULACIÓN DE MICORRIZAS MVA EN PAPA (Solanum tuberosum) RESPUESTA EN EL CRECIMIENTO Y NUTRICIÓN DE PLANTAS INOCULADAS EN INVERNADERO Y EN CAMPO
}

\author{
PATRICIA MORENO DÍAZ*
}

\section{RESUMEN}

Se estudió la respuesta a la inoculación de micorrizas MVA en papa, utilizando plantas provenientes de tubérculos y de esquejes de tallo enraizados, en ensayos de invernadero y campo respectivamente. De tres especies de hongos micorrizógenos inoculados, sólo Glomus fasciculatum "E3" se asoció con el cultivo infectando las raíces en un 33\% y dando lugar a incrementos significativos en el peso seco de tubérculos y peso seco total, bajo condiciones de invernadero. Plántulas de esquejes de las variedades Mariva y Tomasa Condemayta fueron inoculadas con G. fasciculatum "E3" y transplantadas a camas de almácigos con suelo esterilizado, y al campo. El suelo para ambos contenía 15 ppm de fósforo disponible, $\mathrm{pH} 7.8$ y textura franco-arenosa. Los resultados en almácigos mostraron una respuesta favorable a la inoculación desde el inicio de la estación de crecimiento, siendo los beneficios mayores para la variedad Tomasa Condemayta. Las plantas inoculadas de ambas variedades registraron incrementos altamente significativos respecto a las no inoculadas, en peso seco total, rendimiento de tubérculos y absorción de nutrientes a los 68 días del transplante. Los beneficios en el crecimiento de estas plantas estuvieron en relación directa con los niveles de infección determinados en raíces. En los esquejes inoculados llevados al campo, no se observaron efectos positivos en el crecimiento ni en la absorción de nutrientes.

Palabras Claves Adicionales: Micorrizógenos, esquejes, almácigos, franco-arenosa, peso seco.

\section{VAM Mycorhizal Inoculation into Potato (Solanum tuberosum) Greenhouse and in the Field Response and Growth of Plants Inoculated.}

\begin{abstract}
The response of potato plants to VAM inoculation was studied using plats produced from tubers and cuttings and tested under greenhouse and field conditions. Three species of mycorrhizal fungi were tested, but only Glomus fasciculatum "E3" was found associated with the crop infecting 33\% of the root system. This association produced a significant increase of tubers dry weight and total dry weight at greenhouse conditions. Mariva and Tomasa Condemayta rooted stem cuttings were inoculated and transplanted
\end{abstract}

\footnotetext{
* Laboratorio de Micología y Biotecnología, Facultad de Ciencias. Universidad Nacional Agraria. Apartado 456, Lima-Perú.
} 
to seedbeds with sterilized soil. A similar group were planted directly to the field. In both cases, the soil was sandy-loam, $\mathrm{pH} 7.8$ and contained $15 \mathrm{ppm}$ of Phosphorus. Positive response was noted since the beginning of the growing season in the seedbeds, and this effect was greater in T. Condemayta. Inoculated plants of both varieties showed highly significant response in total dry weight, tuber yield and total nutrient content with respect lo noninoculated. The benefits of growth in these plants were in direct relation-ship to the degree of root infection. Non positive results were observed in the inoculated cutting transplanted to the field.

Additional index Words: Stem cuttings, seedbeds, dry weight, Sandy-loam.

Numerosas investigaciones han establecido la ocurrencia de micorrizas VA en papa Gerdemann, (16); Hayman et al. (25); Black y Tinker (9); Schenck y Smith (40). En estudios de Graham y colaboradores (17), se determinó el efecto de la inoculación de Glomus fasciculatum en el incremento del número de tubérculos y en el peso seco de la parte aérea y radical de las plantas. En 1977, Black y Tinker determinaron que la fertilización fosfatada suprimía considerablemente la infección en plantas inoculadas en el campo, mientras que con escasa cantidad de fósforo alcanzaba un $24.2 \%$. Swaminathan y Verma (42), observaron que Glomus macrocarpus podía promover al cultivo en la asimilación de hasta 8 veces más cantidad de fósforo con el consecutivo incremento en la producción de materia seca. Se han reportado diferentes susceptibilidades a la infección de hongos MVA en cultivares de papa. Entre ellas, un rango de infección de 2 a 55\% para 35 cultivares Montierro y Susana (33) y colonización temprana o tardía en 3 cultivares resistentes al tizón tardío Bhattarai y Mishra (8).

La papa es un cultivo que presenta normalmente un alto requerimiento en fósforo por su escaso sistema radical, período vegetativo corto y una limitada asociación natural con hongos MVA Swaminathan y Verma (44); Montierro y Vander Zaag (34). En la producción de papa no tradicional a partir de esquejes de tallo enraizados, se ha comprobado una demanda mayor de nutrientes y una marcada respuesta en el crecimiento con la fertilización fosfatada, especialmente si se desarrollan en sustratos esterilizados Villagarcía et al. (44); Montierro y Vander Zaag (34). La inoculación de hongos MVA en papa puede resultar de gran beneficio al reducir estos requerimientos y mejorar el equilibrio hídrico y hormonal de las plantas al transplante, así como al hacer más efectiva la fertilización fosfatada.

El objetivo del presente trabajo fue determinar los efectos de la inoculación de micorrizas VA en el crecimiento y estado nutricional de plantas de papa en condiciones de invernadero y campo. 


\section{MATERIALES Y MÉTODOS}

\section{Experimento de Invernadero}

Este experimento se condujo utilizando como semilla, tubérculos del clon DTO-33 ${ }^{(1)}$ que fueron desinfectados superficialmente con hipoclorito de calcio al 2\%. El sustrato de crecimiento consistió en una mezcla esterilizada distribuida en macetas de $5 \mathrm{~kg}$, preparada en proporciones de volumen con base en musgo (1), arena (2), vermiculita (2) y suelo (5). Las características de la mezcla fueron textura franco arenosa, $\mathrm{pH} 7.4$, contenido bajo en nitrógeno y medio en fósforo disponible (7 ppm, Olsen modificado). Se adicionó fertilizante químico a razón de 50 ppm de nitrógeno, fósforo y potasio en forma de nitrato de amonio, superfosfato de calcio triple y sulfato de potasio, respectivamente. Parte de la mezcla no fue esterilizada para realizar una prueba de control complementaria.

El inoculo utilizado por macetas consistió en $100 \mathrm{~g}$ de suelo y estructuras fúngicas de cada una de tres especies de hongos micorrizógenos: Glomus caledonius ${ }^{(2)}$, Glomus mosseae ${ }^{(3)}$, y Glomus fasciculatum "E3"(3). Los controles fueron preparados usando el mismo inoculo puesto en autoclave. Antes de la siembra las macetas recibieron $100 \mathrm{ml}$ de un filtrado de suelo carente de estructuras de hongos MVA reinoculando parte de los microorganismos perdidos durante la esterilización.

El experimento fue instalado siguiendo un diseño estadístico completamente al azar. La cosecha se efectuó en dos etapas, a los 80 y 100 días evaluándose los principales parámetros de crecimiento. Se determinó el contenido de nutrientes (N, P, K) en hojas según Chapman (11). Las raíces fueron clarificadas y teñidas para la observación de la infección siguiendo la técnica de Phillips y Hayman (36). El porcentaje de infección fue determinado haciendo una modificación del método de Giovanetti y Mosse (15). Las raíces teñidas fueron cortadas en segmentos de aproximadamente 2 cm y colocadas ordenadamente sobre láminas portaobjetos previamente trazadas con líneas paralelas. Se evaluaron las intersecciones de las líneas con 100 segmentos de raíz por muestra.

\section{Experimento de Almácigos}

Se utilizaron esquejes de tallos enraizados de dos variedades, Mariva y Tomasa Condemayta. El sustrato, suelo esterilizado de textura franca, pH 7.8 y 15 ppm de fósforo disponible (olsen modificado), fue distribuido en seis almácigos de $1 \mathrm{~m}^{2}$ de área y $25 \mathrm{~cm}$ de profundidad. Se adicionó

${ }^{1}$ Clon producido por el Centro Internacional de la Papa. Perú.

${ }^{2}$ Aislamiento proporcionado por CEPLAC Bahía-Brasil, (Dr. Fernando Ezeta).

3 Aislamientos proporcionados por la Estación Experimental de Rothans-ted. Inglaterra, (Dr. David Hayman) . 
fertilizante químico a razón de 50 ppm de N, P y K además de un filtrado de suelo para restituir parte de la flora microbiana natural. Los esquejes de 15 días de enraizados, fueron inoculados colocándolos en contenedores de tierra vegetal con $150 \mathrm{~g}$ de inoculo consistente en suelo, raíces infectadas, micelio y esporas de Glomus fasciculatum "E3". Para los controles se usó inoculo autoclavado. Pasados 15 días, los esquejes se transplantaron a los almácigos con un distanciamiento de $15 \mathrm{~cm}$.

El diseño estadístico para este experimento fue el completamente randomizado con arreglo factorial. A partir de los 30 días del transplante se realizaron cuatro evaluaciones del crecimiento y del grado de infección en raíces. Se determinó además el contenido de nutrientes $(\mathrm{N}, \mathrm{P}, \mathrm{K})$ en hojas, tallos y tubérculos según Chapman (11).

\section{Experimento de Campo}

Este experimento se condujo paralelamente al de almácigos usando el campo experimental de donde se extrajo el suelo de aquellos, el mismo material vegetal y similar inoculo. Fueron transplantados 600 esquejes en un área de $646 \mathrm{~m}^{2}$. La aplicación de fertilizantes se realizó al fondo del surco y lateral a cada planta en una dosis de N-P-K (50-50-50). El diseño experimental fue parcelas divididas con arreglo factorial y dispuesto en cuatro bloques. Se hicieron tres evaluaciones del crecimiento y de la infección en raíces determinándose además el contenido de nutrientes en hojas, tallos y tubérculos, según los métodos mencionados anteriormente.

\section{RESULTADOS}

\section{Experimento de Invernadero}

Los resultados mostraron una respuesta favorable a la inoculación de hongos MVA bajo condiciones de invernadero en sustrato esterilizado (SE). Entre los parámetros de crecimiento medidos, se encontraron diferencias altamente significativas entre plantas inoculadas y no inoculadas, tanto para el peso seco de tubérculos como para el peso seco total en plantas de 100 días (Tabla 1). 
TABLA 1. Respuesta en el crecimiento de plantas de papa inoculadas con hongos MVA bajo condiciones de invernadero.

\begin{tabular}{|c|c|c|c|c|c|c|c|c|}
\hline Tratamientos & \multicolumn{2}{|c|}{$\begin{array}{l}\text { Peso seco tubérculos } \\
\text { (g) }\end{array}$} & \multicolumn{2}{|c|}{ Peso seco total } & \multirow[t]{2}{*}{$\mathrm{N}$} & \multirow[t]{2}{*}{$\begin{array}{c}\mathrm{P} \\
\text { (foliar) } \\
\text { mg/maceta }\end{array}$} & \multirow[t]{2}{*}{ K } & \multirow[t]{2}{*}{$\begin{array}{l}\text { Infección en } \\
\text { raíces (\%) }\end{array}$} \\
\hline & $\mathrm{Cl}$ & $\mathrm{C} 2$ & $\mathrm{Cl}$ & $\mathrm{C} 2$ & & & & \\
\hline G. caledonius & 8.9 & 15.3 & 16.6 & 22.3 & 609 & 49 & 435 & 4.4 \\
\hline G. mosseae & 9.1 & 15.7 & 17.2 & S'3.5 & 609 & 49 & 329 & 6.5 \\
\hline G. fasciculatum "E3" & 8.6 & 19.1 & 17.9 & 27.8 & 881 & 67 & 423 & 33.2 \\
\hline \multirow{4}{*}{$\begin{array}{l}\text { Control SNE } \\
\text { Control SE } \\
\text { Signif. estadist. } \\
\text { DLS } 0.5\end{array}$} & 6.1 & 16.6 & 15.4 & 26.1 & 812 & 52 & 416 & 18.1 \\
\hline & 5.3 & 15.7 & 12.4 & 24.2 & 640 & 48 & 341 & - \\
\hline & n.s & ** & n.s & $* *$ & * & * & * & $* *$ \\
\hline & & 1.8 & & 2.2 & 134.6 & 11.9 & 106.4 & 7.4 \\
\hline \multirow{2}{*}{\multicolumn{9}{|c|}{$\begin{array}{ll}\text { ns } & \text { no significativo } \\
* & \text { significativo al } 0.05 \%\end{array}$}} \\
\hline & & & & & & & & \\
\hline \multicolumn{9}{|c|}{$* * \quad$ significativo al $0.01 \%$} \\
\hline \multicolumn{9}{|c|}{$\begin{array}{ll}\text { SNE } & \text { sustrato no esterilizado } \\
\text { SE } & \text { sustrato esterilizado }\end{array}$} \\
\hline \multicolumn{9}{|c|}{ SE $\quad$ sustrato esterilizado } \\
\hline \multicolumn{9}{|c|}{ C1 1ra.cosecha a los 80 días. } \\
\hline \multicolumn{9}{|c|}{ C2 2da. cosecha a los 100 días. } \\
\hline Peso seco de & a parte aérea & & & & & & & \\
\hline
\end{tabular}


De las tres especies inoculadas, G. fasciculatum "E" fue la que otorgó mayores beneficios en el crecimiento de las plantas en comparación a las no inoculadas (Figura 1). Los niveles más altos en nutrientes absorbidos $\mathrm{N}$ y $\mathrm{P}$ (mg/maceta), se encontraron asimismo en las plantas inoculadas con $G$. fasciculatum " $\mathrm{E}_{3} "$, siendo las diferencias estadísticamente significativas respecto a los controles. Para potasio el valor más alto en absorción fue obtenido con G. caledonius.

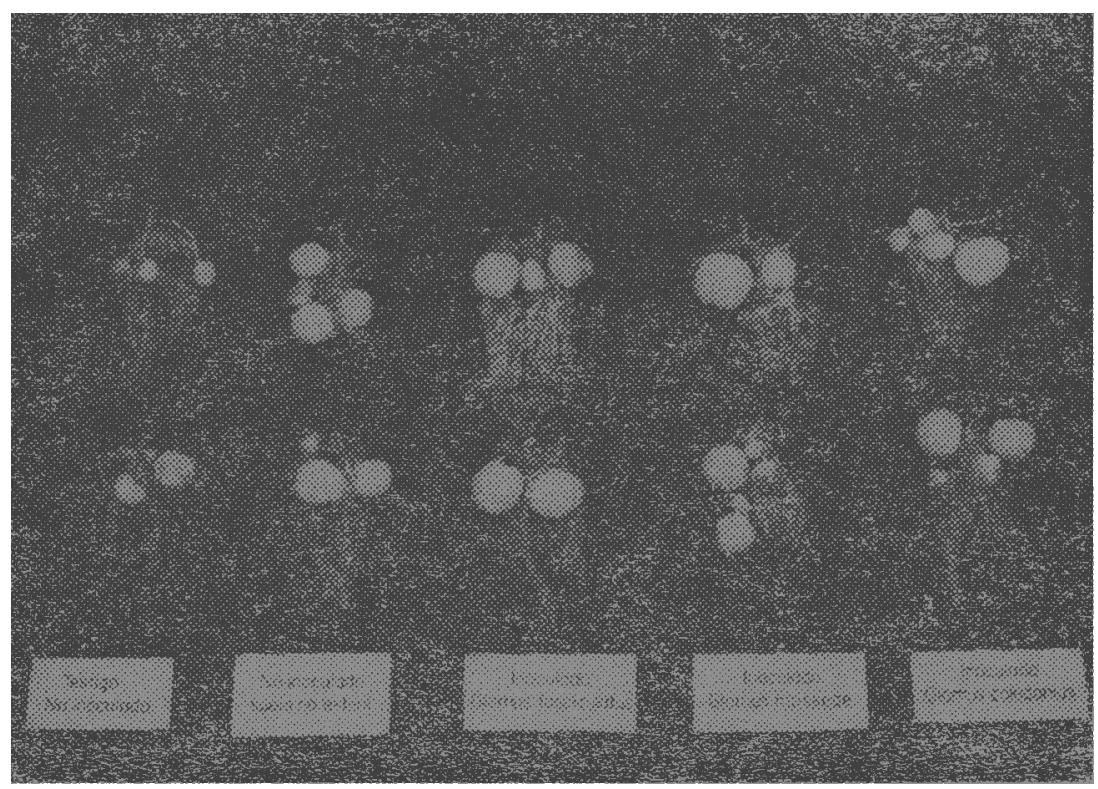

FIGURA 1 Rendimiento de tubérculos en macetas de papa inoculadas con especies micorrizógenas.

Las plantas de los tratamientos inoculados no superaron estadísticamente en contenido de nutrientes a las plantas no inoculadas crecidas en sustrato no esterilizado (Control SNE). Observando el porcentaje de infección en raíces, G. fasciculatum " $\mathrm{E}_{3}$ " aparece como la especie más infectiva de las inoculadas con un 33\% de raíces colonizadas, lo cual estuvo en relación directa con los efectos observados en el crecimiento. Mientras G. mosseae y G. caledonius infectaron muy débilmente el cultivo, en el suelo natural del sustrato no esterilizado (SNE), la existencia de una población nativa de hongos MVA logró infectar las raíces en un $18 \%$ con algunos efectos positivos en el crecimiento de las plantas (Tabla 1). 

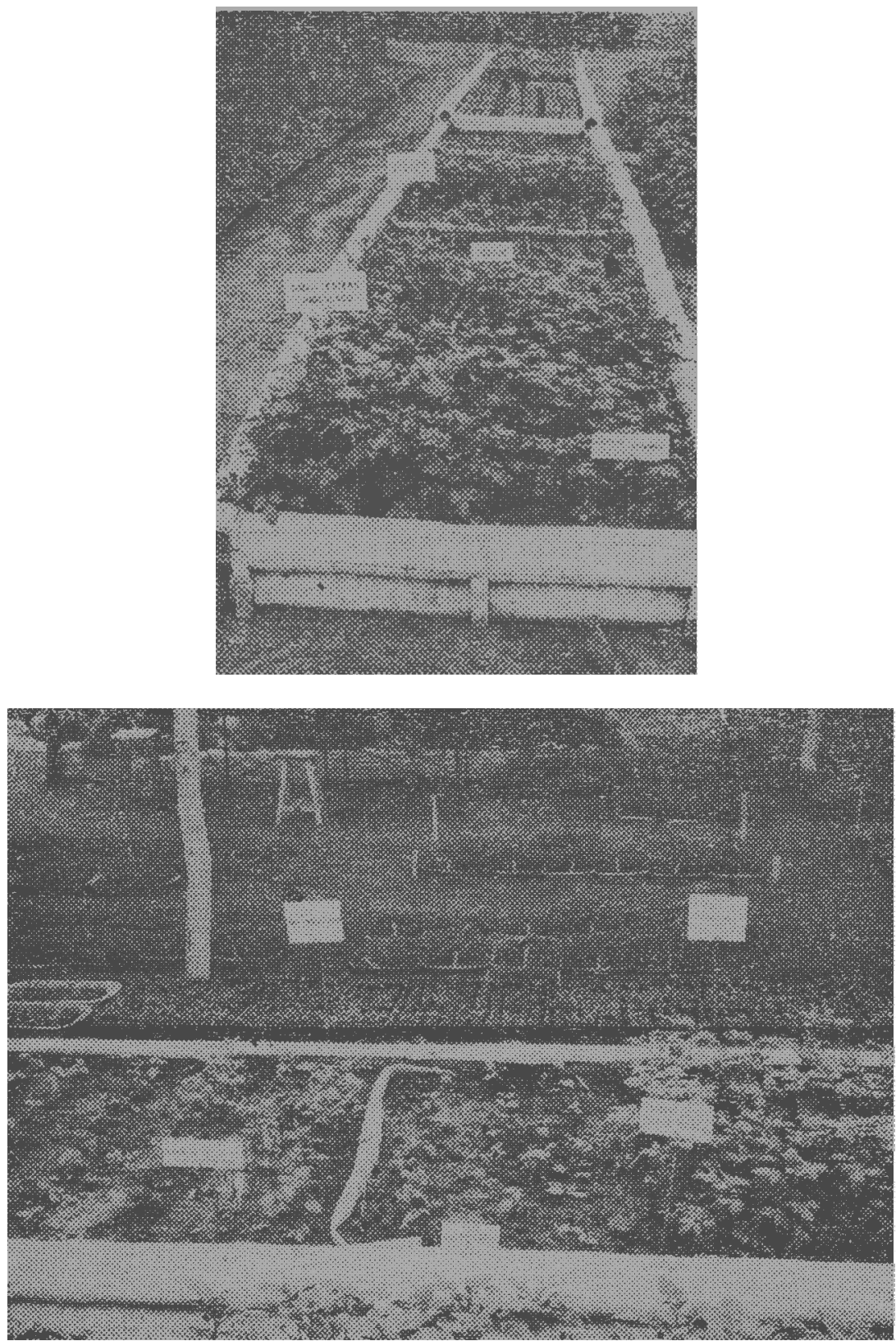

FIGURA 2. Respuesta a la inoculación de Glomus fasciculatum "ES" en plantas de papa provenientes de esquejes y desarrolladas en almácigos. (a) Al frente inoculado, al fondo no inoculado (b) A la izquierda no inoculado, a la derecha inoculado. 


\section{Experimento de Almácigos}

Los esquejes inoculados desarrollados en almácigos mostraron desde el inicio un mejor estado de crecimiento y mayor vigor que los no inoculados, permaneciendo con estas diferencias hasta el fin del período de crecimiento (Fig. 2). A los 68 días del transplante se determinaron diferencias altamente significativas en el rendimiento de tubérculos y en el peso seco total de las dos variedades inoculadas, duplicando o triplicando los valores obtenidos en las plantas no inoculadas (Tabla 2). La tendencia seguida en la ganancia de peso a través del período de crecimiento en plantas inoculadas y no inoculadas se observa en la Figura 3.

TABLA 2. Respuesta a la inoculación de G. fasciculatum "E3" en esquejes de las variedades Mariva (M) y Tomasa Condemayta (T. C.) a los 68 días del transplante en almácigo.

\begin{tabular}{|c|c|c|c|c|c|c|}
\hline \multirow[t]{2}{*}{ Tratamientos } & \multicolumn{2}{|c|}{$\begin{array}{l}\text { Rendimiento de tubérculos } \\
\text { (g/planta) }\end{array}$} & \multicolumn{2}{|c|}{$\begin{array}{l}\text { Peso Seco } \\
\text { total }\end{array}$} & \multicolumn{2}{|c|}{$\begin{array}{l}\text { Infección en } \\
\text { raíces } \\
(\%)\end{array}$} \\
\hline & $\mathrm{M}$ & T.C. & $\mathrm{M}$ & T.C. & M & T.C \\
\hline Inoculadas & 77.2 & 119.6 & 22.2 & 46.7 & 41.8 & 58.0 \\
\hline No Inoculadas & 40.1 & 32.7 & 15.1 & 13.8 & -- & -- \\
\hline $\begin{array}{l}\text { Signif. } \\
\text { estadística de la } \\
\text { interacción }\end{array}$ & & & & & & \\
\hline
\end{tabular}

Las plantas no inoculadas de Tomasa Condemayta se mostraron débiles desde el transplante y no sobrevivieron a la última evaluación. Se encontró una respuesta diferencial entre las variedades de papa estudiadas. Las plantas inoculadas de Tomasa Condemayta superaron a las de Mariva, mostrando mayores diferencias en crecimiento respecto a las no inoculadas a través de toda la estación de cultivo. Esto tuvo relación directa con los niveles de infección determinados en raíces, T. Condemayta se infectó en 58\% a los 68 días con un valor promedio de $66 \%$ en las cuatro evaluaciones realizadas; Mariva entretanto, registró un $41.8 \%$ a los 68 días y $45 \%$ en promedio. Los niveles más altos de infección se obtuvieron a los 30 días después del transplante $\mathrm{y}$ en las etapas siguientes, los valores no variaron considerablemente.

En cuanto a la cantidad de nutrientes absorbidos, se encontraron diferencias significativas entre plantas inoculadas y no inoculadas de $\mathrm{T}$. Condemayta en la absorción de fósforo y potasio a los 91 días después del transplante (TABLA 3). 


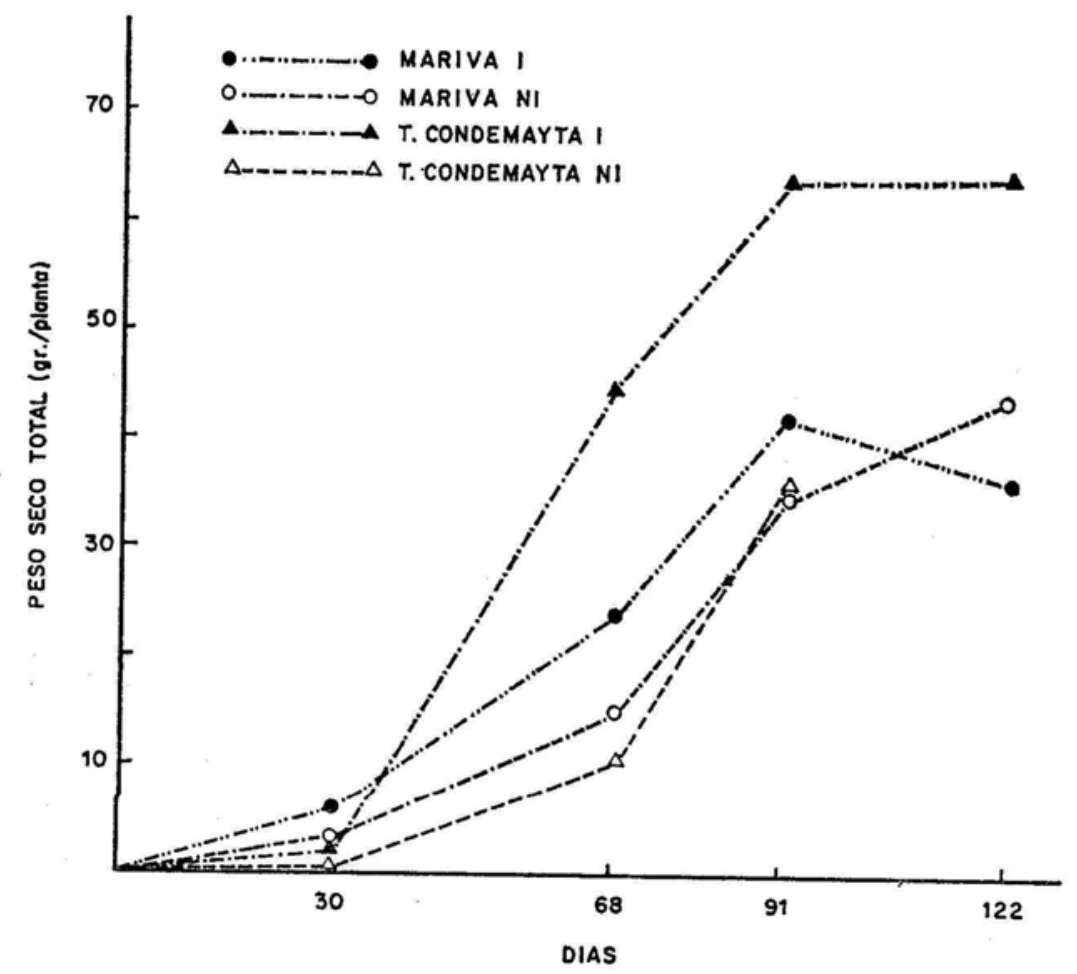

FIGURA 3. Peso seco total obtenido en almácigos con dos variedades de papa inoculada (I) y no inoculada (NI) durante el período de crecimiento.

TABLA 3. Contenido total ${ }^{1}$ promedio de nutrientes en plantas inoculadas con $G$. fasciculatum "E3" a los 91 días después del transplante en almácigos.

\begin{tabular}{|c|c|c|c|c|c|c|}
\hline & $\%$ & $\begin{array}{c}\mathrm{N} \\
\mathrm{mg} / \text { planta }\end{array}$ & $\%$ & $\begin{array}{c}\mathrm{P} \\
\text { mg/planta }\end{array}$ & $\%$ & $\begin{array}{c}\mathrm{K} \\
\mathrm{mg} / \text { planta }\end{array}$ \\
\hline \multicolumn{7}{|l|}{ Mariva } \\
\hline Inoculado & $1.9 \mathrm{a}$ & $837 \mathrm{ab}$ & $0.3 \mathrm{a}$ & $140 \mathrm{ab}$ & $2.3 \mathrm{~b}$ & $991 \mathrm{~b}$ \\
\hline No inoculado & $1.7 \mathrm{ab}$ & 290 b & $0.2 \mathrm{a}$ & $36 \mathrm{~b}$ & $2.0 \mathrm{~b}$ & $339 \mathrm{~b}$ \\
\hline T. Condemayta & & & & & & \\
\hline Inoculado & $1.4 \mathrm{ab}$ & 107 a & $0.2 \mathrm{a}$ & 184 a & $2.5 \mathrm{a}$ & 1876 a \\
\hline No Inoculado & $1.8 \mathrm{a}$ & $525 a b$ & $0.2 \mathrm{a}$ & $60 \mathrm{~b}$ & $2.3 \mathrm{~b}$ & $669 \mathrm{~b}$ \\
\hline
\end{tabular}

1 Incluye hojas, tallos y tubérculos.

(Letras similares no difieren estadísticamente al $0.05 \%$ prueba de Duncan). 
En la absorción de nitrógeno, fósforo y potasio, los puntos de máxima absorción en plantas inoculadas de ambas variedades se alcanzaron entre los 90 y 120 días. Sin embargo, los incrementos en la absorción fueron notables respecto a las plantas no inoculadas desde los 30 días de la inoculación. Los valores de extracción finales de los tratamientos permiten apreciar estas diferencias (Figs. 4, 5 y 6).

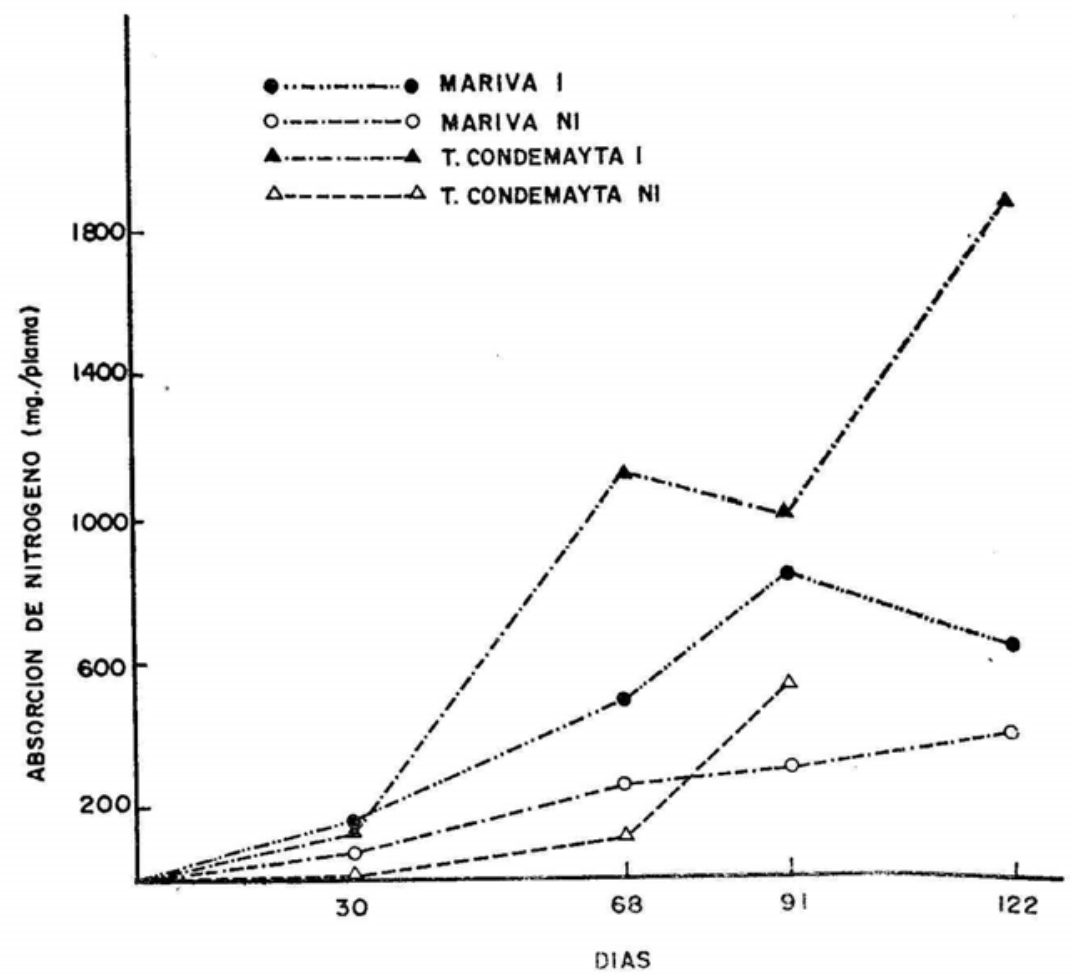

FIGURA 4. Nitrógeno total absorbido en plantas inoculadas (I) y no inoculadas (NI) desarrolladas en almácigos, durante el periodo de crecimiento. 

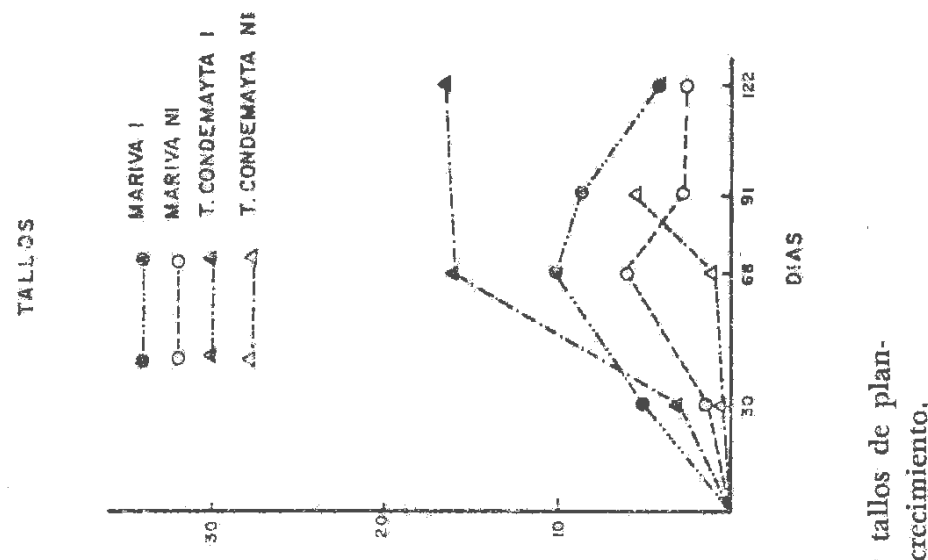

(tow) Oyos sos 30 Nolouosgy

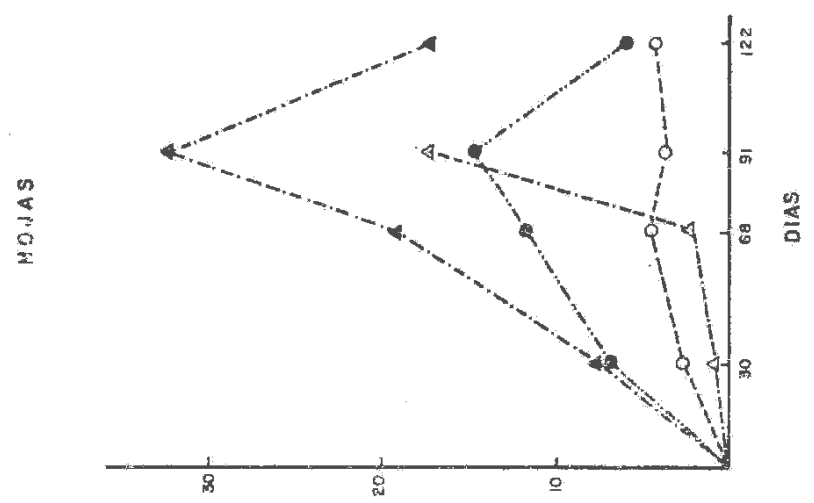

월

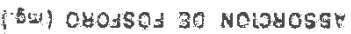

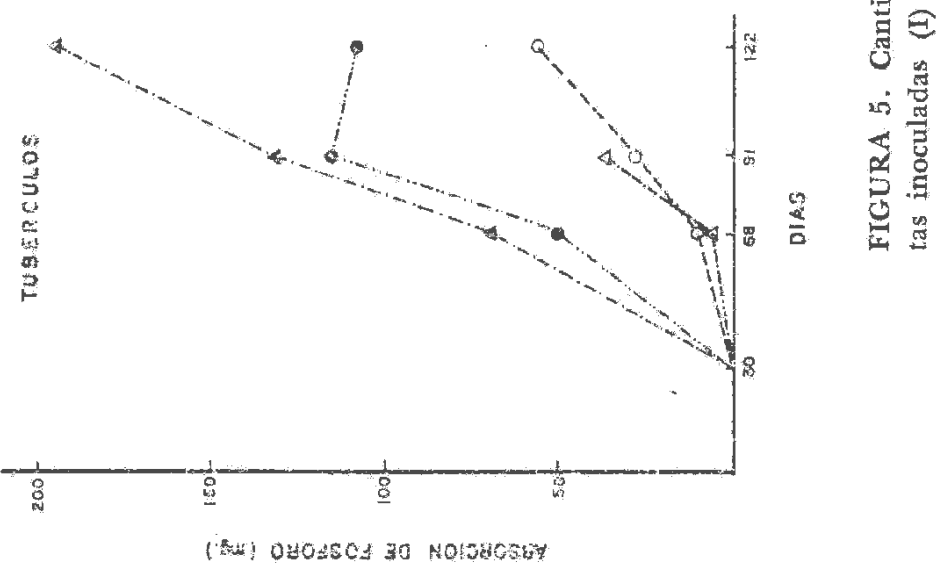




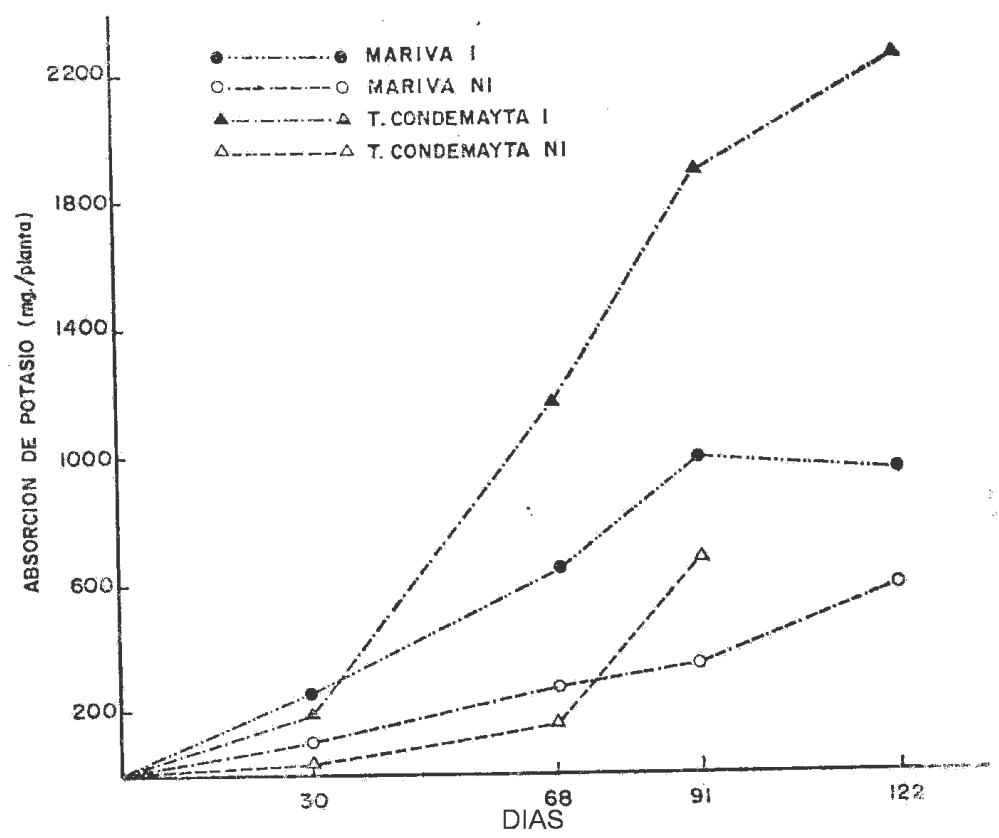

FIGURA 6. Potasio total absorbido en plantas inoculadas (I) y no inoculadas (NI) desarrolladas en almácigos, durante el período de crecimiento.

Las plantas inoculadas de T. Condemayta absorbieron mayor cantidad de fósforo en hojas, tallos y tubérculos respecto a Mariva, y las diferencias con las plantas no inoculadas también fueron mayores con la primera. Las cantidades de fósforo absorbidas en hojas y tallos tuvieron un máximo entre los 60 y 91 días, mientras que en tubérculos se incrementaron hasta el final del período de cultivo (Fig. 5).

\section{Experimento de Campo}

En la primera etapa del desarrollo de los esquejes en el campo, se observó una pérdida considerable por efecto del transplante que fue más intensa en los esquejes no inoculados de T. Condemayta. El porcentaje de sobrevivencia determinado en plantas inoculadas y no inoculadas fue de 84 y $73 \%$ en Mariva y de 78 y 49\% en T. Condemayta.

Los resultados de las dos evaluaciones del crecimiento mostraron que a los 68 días del transplante no hubo diferencias significativas en el rendimiento de tubérculos ni en el peso seco total en plantas con y sin inoculación. Esto permaneció con la misma tendencia hasta 92 días del 
transplante para T. Condemayta, mientras que para Mariva se observaron los mismos parámetros favorables a las plantas no inoculadas.

La determinación del porcentaje de infección en raíces demostró la presencia de una población de hongos micorrizógenos nativos que infectaron las plantas no inoculadas y que probablemente compitieron negativamente con la cepa inoculada (Tabla 4)

En la absorción de nutrientes ( $\mathrm{N}, \mathrm{P}$ y $\mathrm{K}$ ) no se encontraron diferencias significativas entre plantas inoculadas y no inoculadas a nivel de hojas, tallos y tubérculos.

TABLA 4. Respuesta a la inoculación de G. fasciculatum "E3" en esquejes de las variedades Mariva (M) y Tomasa Condemayta (T. C.) desarrollados en el campo.

\begin{tabular}{|c|c|c|c|c|c|c|}
\hline \multirow{2}{*}{$\begin{array}{l}\text { Tratamientos } \\
68 \text { días }\end{array}$} & \multicolumn{2}{|c|}{$\begin{array}{c}\text { Peso fresco tubérculos } \\
\text { g/planta }\end{array}$} & \multicolumn{2}{|c|}{$\begin{array}{l}\text { Peso seco total } \\
\text { g/planta }\end{array}$} & \multicolumn{2}{|c|}{$\begin{array}{l}\text { Infección en } \\
\text { raíces }\end{array}$} \\
\hline & $\mathrm{M}$ & T.C. & $\mathrm{M}$ & T.C. & $\mathrm{M}$ & T.C. \\
\hline Inoculadas & 145.8 & 204.7 & 47.6 & 58.8 & 37 & 46 \\
\hline No Inoculadas & 214.3 & 151.1 & 76.4 & 51.0 & 17 & 10 \\
\hline $\begin{array}{l}\text { Signif. estadíst. } \\
\text { de la interacción } \\
92 \text { días }\end{array}$ & \multicolumn{2}{|c|}{ n.s } & \multicolumn{2}{|c|}{ n.s } & \multicolumn{2}{|c|}{$* *$} \\
\hline Inoculadas & 551.0 & 503.6 & 138.3 & 142.0 & 24 & 18 \\
\hline No Inoculadas & 712.4 & 459.0 & 208.2 & 130.1 & 10 & 15 \\
\hline $\begin{array}{l}\text { Signif. estadist. } \\
\text { de la interacción }\end{array}$ & \multicolumn{2}{|c|}{$*$} & \multicolumn{2}{|c|}{ * } & \multicolumn{2}{|c|}{$* *$} \\
\hline $\begin{array}{ll}* & \text { significativo } \\
* * & \text { significativo } \\
\text { n.s. } & \text { no significat }\end{array}$ & $\begin{array}{l}\text { al } 0.05 \% \\
\text { al } 0.01 \%\end{array}$ & & & & & \\
\hline
\end{tabular}

\section{DISCUSIÓN}

La respuesta a la inoculación de hongos MVA en papa fue favorable y dependiente de las características de los tres miembros de la simbiosis de acuerdo con Hayman $(23,24) G$. fasciculatum "E ${ }_{3}$ " estableció la mejor asociación con el cultivo entre los endofitos probados en condiciones de invernadero.

G. fasciculatum ha demostrado una marcada eficiencia en muchas especies vegetales inoculadas La Rue et al. (29); Granan et al. (17); Hayman (20, 22); Abbot y Robson (3). Es sabido que los hongos MVA muestran diferencias óptimas de acuerdo con los factores edáficos Green et al. (18); Daniels y Trappe (14); Abbot y Robson (2) y en especial al pH del suelo Hayman y Tavares (26). Se ha reportado que G. fasciculatum "E ${ }_{3}$ " puede establecer una buena asociación en condiciones de suelo ácido y neutro 
Azcón et al (5); Hayman y Tavares (26) y hasta en pH 7,6 Abbot y Robson (3).

De acuerdo con los resultados obtenidos las condiciones de $\mathrm{pH}$ en el suelo de los experimentos de invernadero y almácigos, permitieron un buen desarrollo de la simbiosis y beneficios para las plantas, al parecer, por la gran capacidad de adaptación del hongo MVA y por la homogeneidad del sustrato. Mientras tanto, los valores de $\mathrm{pH}$ en el campo fueron poco constantes, pudiendo esto haber influenciado negativamente en la operatividad del micelio extramétrico Hayman y Tavares (26).

En cuanto al contenido de fósforo en el suelo, los niveles utilizados parecieron elevados respecto al trabajo de Montierro y Vander Zaag (33). Ellos observaron que la infección en esquejes declinó en altos niveles de P, obteniendo los valores más altos con 0 y $50 \mathrm{ug} / \mathrm{g}$ de suelo. Probando 7 niveles de fósforo en el campo, obtuvieron incrementos significativos en el rendimiento de tubérculos con 3.7 ppm y una respuesta positiva hasta con 27.3 ppm de $\mathrm{P}$ en el suelo. Cabe suponer que en condiciones de real escasez del elemento, la respuesta a la inoculación en las pruebas realizadas, hubiera sido más notable, aún en condiciones de campo.

Los esquejes de papa enraizados usados en los experimentos de almácigo y campo demostraron una aparente dependencia a la micorrización para el crecimiento. Esto se explica porque el alto requerimiento de fósforo característico del cultivo aumenta en aquellas plantas que no desarrollan a partir de un tubérculo madre, constituyéndose en un elemento esencial para las primeras semanas del desarrollo Wiersema (45). Considerando que los esquejes inoculados se infectaron tempranamente, la mayor sobrevivencia y tolerancia a los efectos negativos del transplante en almácigos y campo se atribuyó a una respuesta influenciada por la micorrización por tratarse de plantas en mejor estado general de nutrientes.

Los niveles de infección determinados en papa variaron en los tres experimentos desarrollados de acuerdo con las condiciones implementadas. Otros trabajos en este cultivo han reportado también un rango variable de infección en raíces Black y Tinker (9); Swaminathan y Verma (42); Montierro y Susana (33); Montierro y Vander Zaag (34) llegando a valores muy altos (70-80\%) en condiciones de escaso fósforo inicialmente en el suelo Montierro y Vander Zaag (34).

Bajo condiciones de invernadero y en almácigos el mayor porcentaje de infección en raíces generalmente estuvo en relación directa con los efectos benéficos observados en el crecimiento y nutrición de las plantas, como respuesta a la inoculación. Sin embargo, tratándose de suelo natural en el campo con una población nativa de hongos micorrizógenos, esta relación no se cumplió.

Aunque muchos autores refieren que la eficiencia en la toma de fósforo y el beneficio para la planta depende de la extensión y rapidez de la micorrización Tinker (43); Sanders et al (39); Abbot y Robson (1), es más apropiado considerar en nuestro caso que no siempre la infectividad del hongo repercute en efectividad para la planta Hayman (22); Ascon Aguilar et 
al (6), sobre todo en casos de competencia de endofitos. La existencia en el suelo de una población nativa de hongos micorrízicos en muchos casos no puede ser detectada a través de una simple cuantificación de esporas Hall (19) ; Powell (37); Sparling y Tinker (41); Abbot y Robson (1).

En el campo, uno de los más distintivos hongos MVA nativos es el "endofito fino" (Glomus tenuis), el cual ha sido reportado en muchas partes del mundo Crush (12); Daft y Nicolson (13); Abbot y Robson (1). En pruebas de interacción con $G$. fasciculatum sobre trébol subterráneo $G$. tenuis resultó ser menos infectivo pero más agresivo y capaz de excluir a su competidor de las raíces Wilson (47). Se ha demostrado que G. tenuis se extiende más rápidamente de planta a planta que otros endofitos Powell (38), la infección es tardía y se distribuye en la raíz produciendo muchas pequeñas unidades de infección Wilson y Trinick (48); Wilson (46).

Por otro lado Glomus fasciculatum ha sido señalada como especie de alta infectividad para muchos cultivos Hayman (21); Abbot et al, (1984) Wilson $(46,47)$, la infección ocurre en etapa temprana y se distribuye extensivamente dentro de la raíz produciendo infección densa por punto de entrada. Suponiendo que en nuestro experimento el endofito indígeno dominante hubiera iniciado lentamente la infección y desarrollara baja infectividad, esto habría dado oportunidad a que la infección ya iniciada por G. fasciculatum " $\mathrm{E}_{3}$ ", fuera beneficiosa en la primera etapa del crecimiento. Sin embargo, si la cepa indígena dominante (probablemente G. tenuis), resultó ser más agresiva y más ampliamente dispersada en el suelo, su presencia necesariamente pudo obstaculizar el éxito posterior de la inoculación. Además según Wilson (47), la infección total de una colonización mixta no es mayor que la máxima alcanzada por cada hongo aisladamente.

Se obtuvieron diferencias en la respuesta a la inoculación, entre las variedades estudiadas y el Clon DTO-33. Mariva y T. Conde-mayta son dos variedades que presentan una gran adaptabilidad de crecimiento el mismo período vegetativo (semitardío), y un rendimiento muy similar López et al. (31). El Clon DTO-33 es precoz, bien adaptado, de buen rendimiento y homogéneo en su producción. En general los genotipos de plantas pueden variar en el grado de colonización alcanzado, aún con similares tasas de crecimiento de raíces Krishna et al. (28).

La variación genotípica en la colonización y respuesta a las micorrizas puede ser atribuida a una interacción entre el genotipo del hospedero y las preferencias de la cepa de micorrizas VA Mosse (35), al número de sitios de infección y a las tasas de crecimiento del hongo a través de la corteza Wilson (46). Esto último estaría relacionado con el balance de sustancias inhibidoras producidas por las plantas en respuesta a la infección, como ac. fenólicos, fitoalexinas y otras promotoras como los carbohidratos Krishna et al, (28).

Una alternativa para interpretar estas diferencias sería el determinar la concentración de fósforo a nivel de raíces, valor que puede ser variable entre los genotipos de plantas Clark (10), y que indicaría la tasa posible de colonización en cada caso Jasper et al, (27); Lopes et al. (30); Barea et al. (7); Hayman (24). 
La diferencia de susceptibilidad a la colonización en cultivares de papa también fue reportada por Montierro y Susana (33). Ellas indicaron que la infección ocurre tarde en la estación de crecimiento y que los niveles alcanzados generalmente son bajos. Según Smith, citado por Macedo (32), la diferencia en la absorción de nutrientes entre cultivares de maduración precoz requieren de mayor cantidad de nutrientes al inicio de crecimiento, mientras que los tardíos, al final. Relacionando la absorción de nutrientes con la infección en raíces, debe considerarse que las variedades semitardías y tardías tendrían mayor beneficio a través de la simbiosis respecto a las variedades precoces, al establecerse una absorción de nutrientes efectiva si la infección ocurre tarde, o más prolongada si ésta es temprana.

\section{CONCLUSIONES}

La papa es un cultivo que puede responder favorablemente a la inoculación de micorrizas VA. El grado de respuesta es variable dependiendo de la especie de hongo involucrada, de la variedad y forma propagativa de la planta y de las características del sustrato.

Las plantas provenientes de esquejes de tallo enraizados, pueden beneficiarse en mayor grado con la micorrización por su alta demanda de fósforo desde los inicios del período de crecimiento.

La respuesta a la inoculación será mayor mientras más grande sea la limitación de fósforo disponible en el suelo.

Glomus fasciculatum "E3" es capaz de establecer una buena asociación con el cultivo de papa. Las raíces pueden ser intensamente infectadas y obtenerse beneficios notorios en el crecimiento y estado nutricional de plantas inoculadas, ya sea provenientes de tubérculos o de esquejes enraizados.

Las diferencias en el grado de colonización y respuesta en el crecimiento observadas en Mariva y Tomasa Condemayta, indican la existencia de factores genéticos inherentes a la planta que deben ser considerados en una posterior selección de cultivares micotróficos.

La inoculación de G. fasciculatum "E3" en esquejes que se desarrollaron en el campo no fue efectiva. Se consideran como causas de este efecto: los valores altos de fósforo en el suelo, $\mathrm{pH}$ variable y el factor competitivo de la población de endofitos nativos en el mismo.

\section{Agradecimiento}

Agradezco al doctor David Midmore y al doctor Fernando N. Ezeta del Centro Internacional de la Papa y Programa Nacional de Papa respectivamente, por la valiosa ayuda prestada en la ejecución de este trabajo. 


\section{REFERENCIAS BIBLIOGRÁFICAS}

1. Abbot, L. K.; Robson, A. D. 1982. Infectivity of vesicular-arbuscular fungi in agricultural soils. Aust. J. Agric. Res. 33: 1049-59.

2. Abbot, L. K.; Robson, A. D. 1984. The effect of root density, inoculum placement and infectivity of inoculum on the development of vesicular-arbuscular mycorrhizas. New Phytologist 97: 285-299.

3. Abbot, L. K.; Robson, A. D. 1985. The effect of soil pH on the formation of VA mycorrhizas by two especies of Glomus. Aust. J. Scient. Res. 23: 253-261.

4. Abbot, L. K.; Robson, A. D.; G. de Boer, 1984. The effect of phosphourus on the formation of hyphae in soil by the vesicular arbuscular mycorrhizal fungus Glomus fasciculatum. New Phytologist 97; 437-446.

5. Azcon, R.; Barea; J. M.; Hayman, D. S. 1976. Utilization of rock phosphate in alkaline soils by plants inoculated with mycorrhizal fungi and phospathe-solubilizing bacteria. Soil Biol. Biochem 8: 135-138.

6. Azcon-Aguilar, C.; Barea, J. M.; Roldán-Fajardo, B. 1984. Avances recientes en el estudio de las micorrizas VA II. Factores que afectan su formación función y aplicaciones prácticas en agricultura (1). Anales de Edafología y Agrobiología tomo XLIII N 5-6: 943-958.

7. Barea, J. M.; Azcon-Aguilar, C.; Roldán-Fajardo, B. 1984. Avances recientes en el estudio de las micorrizas VA I. Formación, funcionamiento y efectos en nutrición vegetal (1). Anales de Edafología y Agrobiología Tomo XLIII N³ 3-4: 659-677.

8. Bhattarai, J. D.; Mishra, R. R. 1984. Study on the vesicular-arbuscular mycorrhiza of cultivars of potato (Solanum tuberosum L.). Plant and Soil. 79: 299-303.

9 Black, R. L. B.; Tinker, P. B. 1977. Interactions between effect of vesicular-arbuscular mycorrhiza and fertilizer phosphorus on yields of potatoes in the field. Nature. Lond. 265: 510-511.

10. Clarck, R. B. 1983. Plant genotype differences in the uptake, traslocation, acumulation, and use of mineral elements required for plant growth. Plant and Soil 72: 175-196.

11. Chapman, H. 1979. Métodos de Análisis para suelos, plantas y agua. Edit. Trillas, México. 195p.

12. Crush, J. R., 1973. The effect of Rhizophagus tenuis mycorrhizas on ryegrass, cocksfoot and sweett vernal. New Phytologist 72: 965-973.

13. Daft, M. J.; Nicolson, T. H. 1974. Arbuscular mycorrhizas in plants colonizing coal wastes in Scotland. New Phytologist 73: 1129-1138.

14. Daniels, B. A.; Trappe, J. M. 1980. Factors affecting spore germination of the vesicular-arbuscular mycorrhizal fungus Glomus epigaeus. Mycología 72: 457-471. 
15. Giovannetti, M.; Mosse, B. 1980. An evaluation of techniques for measuring vesicular-arbuscular mycorrhizal infections in roots. New Phytologist. 84: 489-500.

16. Gerdeman, J. W. 1968. Vesicular-arbuscular mycorrhiza and plant growth. Ann. Rev. Phytopathol. 6:379-418.

17. Graham, S. O.; Green, N. E.; Hendrix, J. W. 1976. The influence of vesicular-arbuscular mycorrhizal fungí on growth and tuberization of potatoes. Micología. 68: 925-934.

18. Green, N. E.; Graham, S. E.; Schenck, N. C. 1976. The influence of pH on the germination of vesicular-arbuscular mycorrhizal spores. Mycología. 68: 929-934.

19. Hall, I. R. 1977. Species and mycorrhizal infections of New Zealand Endo-gonaceae. Transaction of the British Mycological Society 68: 341-356.

20. Hayman, D. S. 1977. Mycorrhizal effects on white clover in relation to hill land improvement. Agric. Res. Council Res. Rev. 3: 82-85.

21- Hayman, D. S., 1981. Mycorrhizal and its significance in Horticulture. The Plantsman Vol. 2 part. 4: 214-224.

22. Hayman, D. S. 1983. The physiology of vesicular-arbuscular endomycorrhizal symbiosis. Can. J. Bot. 61: 944-963.

23. Hayman, D. S. 1984. Methods for evaluating and manipulating vesicular-arbuscular mycorrhiza. Microbiological Methods for Enviromental Biotecnology ISBN. 0-12-295040-2. London. pp. 95117.

24. Hayman, D. S. 1985. Effect of plant species, soil and enviromental factors on vesicular-arbuscular mycorrhizal (VAM) infection and nutrient uptake. En: Nuclear Techniques to Study the Role of Mycorrhiza in Increassing Food Crop Production. Internacional Atomic Energy Agency. Vienna. Tec. Doc. 338: 29-47.

25. Hayman, D. S.; Johnson, A. M.; Ruddlesdin, J. 1975. The influence of phosphate and crop species on ndogone spores and vesiculararbuscular mycorrhiza under field conditions, Plant and Soil. 43: 489-495.

26. Hayman, D. S.; Tavares, M. 1985. Plant growth responses to vesiculararbuscular mycorrhiza XV. Influence of soil $\mathrm{pH}$ on the symbiotic efficiency of different endophytes. New Phytologist 100: 367-377.

27. Jasper, D. A.; Robson, A. D.; Abbot, L. K. 1979. Phosphorus and the formation of vesicular-arbuscular mycorrhizas. Soil Biol. Biochem. 11: 501-505.

28. Krishna, K. R.; Shetty, K. G. et al., 1985. Genotype dependent variation in mycorrhizal colonization and response to inoculation of pearl millet. Plant and Soil 86: 113-125.

29. La Rue, J. H.; McClellan, W. D.; Peacock, W. L. 1975. Mycorrhizal fungi and peach nursery nutrition, California Agric. 29: 6-7. 
30. Lopes, E. S.; Siqueira, J. O.; Zambolim, L. 1983. Caracterizacao das micorrizas vesiculo-arbusculares (MVA) e seus efeitos no crescimento das plantas. Revista Brasileira de Ciencia do Solo 7: 119.

31. López, C. P.; Egusquiza, R.; Villagomez, V. 1980. El cultivo de la papa. Centro Nacional de Capacitación e Investigación para la Reforma Agraria. CENCIRA. Lima. 197 p.

32. Macedo, M. C. M. 1976. Absorcao de nutrientes por cultivares nacionais de batatinha Solanum tuberosum. Tesis Ing. Agr. Piracicaba, Brasil. 97 p.

33. Montierro, C. G.; Susana, B. 1983. Response of a diverse group of thirty five cultivares to an insothermic environment of the Philipines. International Potato Center. Región VII. Philippines. Working paper 83-11, 8p.

34. Montierro, C.; Vander Zaag. P. 1983. Preliminary results on the status and potential of mycorrhizae in phosphorus nutrition of potatoes Solanum. International Potato Center. Región VII. Philippines. Internal paper 83-5, llp.

35. Mosse, B. 1981. Vesicular-arbuscular mycorrhiza research for tropical agriculture. Hawwaii Institute of Tropical Agriculture and Human Resources. University of Hawaii. Res. Bull. 194.

36. Phillips, J. M.; Hayman, D. S. 1970. Improved procedures for clearing roots and staining parasitic and vesicular-arbuscular mycorrhizal fungi for rapid assesmenl of infection. Trans $\mathrm{Br}$. Mycol. Soc. 55: 158-161.

37. Powell, C. LI. 1977. Mycorrhizas in hill country soils. II. Effect of several mycorrhizal fungi on clover growth in sterilized soils. $\mathrm{N}$. Zeal. J. Agrie. Res. 20: 59-62

38. Powell, C. LI. 1979. Inoculation of white clover and ryegrass seed with mycorrhizal fungi. New Phytologist, 83: 81-85.

39. Sanders, F. E.; Tinker, P. B. et al. 1977. The development of endomycorrhizal root systems. I. Spread of infection and growthpromoling effects with four species of vesicular-arbuscular endophyte. New Phytologist 78: 257-268.

40. Schenck, N. C.; Smith, G. S. 1981. Distribulion and ocurrence of vesicular-arbuscular mycorrhizal fungi on Florida Agricultural Crops. Soil and Crop Science Soviety of Florida. Proceeding 40: 171-175.

41. Sparling G. P.; Tinker, p. B. 1978. Mycorrhizal infection in pennine grass-land. III. Effects of mycorrhizal infection on the growth of white clover. Journal of Applied Ecology 15: 959-964

42. Swaminathan, K.; Verma. B. C. 1977. Symbiotic effect of vesiculararbuscular mycorrhizal fungi on the phosphate nutrition of potatoes. Proc. Indian Acad. Sci. Sect. B. 85: 310-318.

43. Tinker, P. B. 1975. Soil chemistry of phosphorus and mycorrhizal effects on plant growth. En: Sanders, F. E. et al., (eds.). Endomycorrhizas. Academic Press London. pp. 353-372. 
44. Villagarcia, S.; Mayer, R. et al. 1978. Resultados de ensayos de invernadero y de campo sobre fertilización y nutrición mineral en el cultivo de la papa durante el período 1975/1977. Universidad Nacional Agraria La Molina,- Centro Internacional de la Papa. Perú. $62 \mathrm{p}$.

45. Wiersema, S. G. 1984. The production and utilization of seed tubers derived from true Potato seed. Ph. D. Tesis. University of Reading. 1984.

46. Wilson, J. M. 1984a. Competition for infection between vesiculararbuscular mycorrhizal fungi. New Phytologist 97: 427-435.

47. Wilson, J. M. 1984b. Comparative development of infection by three vesicular-arbuscular mycorrhizal fungi. New Phytologist 97: 413426.

48. Wilson, J. M.; Trinick, M. J. 1983. Infection development and interaction between vesicular-arbuscular mycorrhizal fungi. New. Phytologist 93: 543-553. 